\title{
Conditions De Travail Et De Vie Des Enfants Dans Le Secteur Informel A Abidjan : Cas Des Adolescentes Dans Le Petit Commerce
}

\author{
Dr. Coulibaly Doppon Ali \\ Enseignant chercheur à l'UFR Criminologie, Côte d'Ivoire
}

Doi:10.19044/esj.2018.v14n29p170 URL:http://dx.doi.org/10.19044/esj.2018.v14n29p170

\begin{abstract}
This study aims to analyze the working and living conditions of teenagers in the informal sector of the small business in Abidjan. To achieve this goal, the following hypothesis has been formulated: The difficult working and living conditions of adolescent girls in the informal sector favor the development of deviant or marginal behaviors (begging, prostitution, theft, delinquency, violence, etc.). Victimological theories and criminological theories of acting out were used. The methodological approach took into account the documentary research, the inquiry by interview as well as the phenomenological method, the ethnographic method and the dialectic. The study was conducted among 150 respondents. The route technique has been associated with the area technique for the conduct of the field survey. Qualitative and quantitative analysis were used. The results of this research reveal that it is the combination of internal and external factors with households (socio-economic and cultural conditions) that explains the exploitation of children. These factors must be seen simultaneously in the sense that the exploitation of children remains a social and almost structural phenomenon. In this sense, the study reveals the preponderant role of social capital (kinship and other social norms) that has been neglected in the economic analysis of child labor. In fact, by its ambivalence, work participates in material life as well as in social life.
\end{abstract}

Keywords: Child labour, Exploitation, Conditions of households, Social capital, Socio-cultural beliefs.

\section{Resume}

Cette étude a pour objectif d'analyser les conditions de travail et de vie des adolescentes du secteur informel du petit commerce à Abidjan. Pour atteindre cet objectif, l'hypothèse suivante a été formulée : Les conditions de travail et de vie difficiles des adolescentes dans le secteur informel favorisent 
chez elles le développement de comportements déviants ou marginaux (mendicité, prostitution, vol, délinquance, violence, etc.). Les théories victimologiques et les théories criminologiques du passage à l'acte ont été utilisées. La démarche méthodologique a pris en compte la recherche documentaire, l'enquête par entretien ainsi que la méthode phénoménologique, la méthode ethnographique et la dialectique. L'étude a été menée auprès de 150 enquêtés. La technique des itinéraires a été associée à la technique aréolaire pour la conduite de l'enquête sur le terrain. L'analyse qualitative et quantitative ont été exploitées. Les résultats de cette recherche révèlent que c'est la conjugaison de facteurs internes et externes aux ménages (conditions socioéconomiques et culturelles) qui explique l'exploitation des enfants. Ces facteurs doivent être perçus simultanément en ce sens que l'exploitation des enfants reste un phénomène social et presque structurel. Dans ce sens, l'étude révèle le rôle prépondérant du capital social (lien de parenté et autres normes sociales) qui a été négligé dans l'analyse économique du travail des enfants. En fait, par son ambivalence, le travail participe de la vie matérielle aussi bien que de la vie sociale.

Mots clés : Travail des enfants, Exploitation, Condition des ménages, capital social, croyances socioculturelles, secteur informel.

\section{Introduction : Quelques repères théoriques}

La généralisation du travail des enfants a amené la littérature à s'orienter sur leur insertion sociale. Il ressort de certains travaux l'aspect psychosocial qui oriente les ONG et les institutions dans la lutte contre le phénomène. La généralisation du travail des enfants s'accompagne d'une accentuation de l'exploitation de leur travail et d'une augmentation de ses formes extrêmes ${ }^{30}$. Ainsi, dans une étude de Diallo (2001), les facteurs explicatifs de l'offre et de la demande du phénomène sont examinés, les résultats montrent que l'emploi précoce des enfants en Côte d'Ivoire résulte de la conjugaison des facteurs internes et externes au ménage. Il relève également le rôle prépondérant du capital social qui a été négligé dans l'analyse économique de la participation des enfants aux activités socioéconomiques. Pour lui, les facteurs de l'offre du travail sont les normes sociales. L'emploi précoce de l'enfant s'inscrit dans le cadre de l'obligation de se soutenir entre membres d'une même communauté.

Les auteurs vont démontrer que le travail des enfants est éprouvant et est une source de souffrance d'où l'exploitation. Ce travail viole le droit des

30 Cette généralisation est difficile à prouver car les données statistiques ne sont pas comparables d'une étude à l'autre, mais au vu de la prépondérance des motivations économiques dans la mise au travail des enfants, on peut être sûr qu'elle est corrélative à l'augmentation de la pauvreté. 
enfants et porte atteinte à son développement psychosocial, à sa santé et à son instruction. L'exploitation de l'enfant est alors décrite par Bonnassieux (Agbadou, 2009) comme un contrat signé entre la famille, le trafiquant et le planteur.

Le Document de Stratégie de Réduction de la Pauvreté élaboré en 2008 affirme que le nombre de pauvres en Côte d'Ivoire a quintuplé en passant de $10 \%$ en 1985 à 49\% en 2008. Dans un pays dont le tiers de la population vit directement ou indirectement grâce à la production cacaoyère, parmi les différents facteurs de la crise économique, la chute du prix du cacao, surtout du revenu bord-champ perçu par les paysans, jouent un rôle déterminant. C'est ainsi qu'une économie rurale auparavant dynamique traverse un déclin profond qui s'accompagne de conséquences graves sur plusieurs aspects de l'existence du pays, comme l'exploitation du travail des enfants qui a atteint des proportions endémiques, comme l'affirme l'Enquête Nationale sur le Travail des Enfants qui en 2005 a estimé qu'en Côte d'Ivoire, un enfant sur quatre, âgé de 5 à 17 ans travaillait.

Ainsi, la participation des enfants au travail des adultes est une pratique culturelle en racinée et valorisée (Andvig et Alii cité par BM, 2001). Cette participation qui s'effectue le plus souvent, mais pas toujours, dans un cadre familiale, est un élément fondamental de socialisation de ceux-ci. Cette intégration précoce des enfants aux activités domestiques et économiques vise à les préparer à être, dans un futur plus ou moins lointain, des acteurs de pérennisation des sources de revenus de la famille.

Ainsi, dans un environnement socioculturel favorable à l'utilisation de la force du travail des enfants, la réaction face au recours de la main d'œuvre enfantine viendrait des limites rencontrées par des politiques de scolarisation, et surtout de la persistance de la pauvreté (Lachaud, 2004). Dumas (2005) affirme qu'en dépit d'une diminution progressive de l'ampleur du phénomène notamment dans le domaine agricole, le taux de recours à la main d'œuvre enfantine se maintient à des niveaux encore élevés dans le milieu agricole.

De façon de plus en plus générale, le travail des enfants a cessé d'être une transmission des connaissances et du savoir-faire d'un métier entre l'adulte et l'enfant, mais aussi des valeurs et d'éthique du comportement social. Au contraire, ce que les enfants subissent chaque jour c'est l'exploitation de leur travail et l'atteinte à leur dignité individuelle et sociale à travers la réalisation d'activités dommageables qui, au même temps, sont les causes et effets d'autres violations. Par exemple, plus que 40\% (ENV, 2008), des enfants en âge d'aller à l'école ne sont pas scolarisés du fait que la majorité des ménages vit dans la pauvreté et surtout à cause des carences du système scolaire. L'école est devenue moins accessible, plus chère et avec une rentabilité sur le long terme de plus en plus faible et aléatoire. Tous ces éléments amènent les 
membres des familles à des attitudes négatives concernant l'investissement scolaire en général.

D’un autre côté, le déclin dramatique de l'économie rurale a augmenté le nombre d'enfants migrants et surtout empiré les conditions dans lesquelles ces migrations ont lieu. Auparavant, l'envoi des enfants chez des parents qui résidaient en ville représentait une opportunité d'amélioration de la vie des enfants à travers un meilleur accès à des soins de santé et à une éducation qui souvent n'étaient pas disponibles ou satisfaisants dans le village d'origine. Au cours des dernières décennies, la hausse de la pauvreté et de l'instabilité sociopolitique a engendré un affaiblissement généralisé des garanties de protection offertes par l'Etat et surtout par les familles elles-mêmes. De cette façon, le "confiage" aujourd'hui finit souvent par favoriser la violation de plusieurs droits humains fondamentaux comme le droit de l'enfant à être protégé de toutes formes d'abus, négligence, violence et exploitation.

La cause fondamentale de l'exploitation du travail des enfants est économique, en Côte d'Ivoire comme ailleurs. Cette exploitation progresse de pair avec la misère, car elle constitue une stratégie de survie, pour les familles pourvoyeuses d'enfants travailleurs (l'offre), et pour les employeurs d'enfants (la demande). Les adultes, parce qu'ils gagnent insuffisamment leur vie, sont poussés à utiliser la main d'œuvre infantile soit leurs propres enfants ou soit d'autres enfants pour compléter leurs revenus, pour transférer certaines activités tandis qu'ils se réorientent vers d'autres plus rémunératrices, ou pour limiter leurs coûts de production. Plus la famille est pauvre, plus les enfants sont susceptibles de travailler : les ménages dont les enfants travaillent gagnent en moyenne 328.000 FCFA par an, contre 465.000 FCFA pour les ménages dont les enfants ne travaillent pas $^{31}$. La première raison de la mise au travail des enfants est la contribution au revenu familial (60\% des enfants travailleurs) ; la socialisation, c'est-à-dire la volonté de formation, vient loin derrière (26\%). La presque totalité des enfants travailleurs contribuent au revenu du ménage (99\%). (ENV, 2008).

L'Etat Ivoirien a renforcé son arsenal juridique en ratifiant les conventions 138 et 182 de l'Organisation Internationale du Travail relatives respectivement à l'âge minimum d'admission à l'emploi et à l'élimination des pires formes de travail infantile. Afin que le système juridique progresse de façon adéquate à ces propos, le gouvernement devra adopter et appliquer l'avant-projet de loi sur la traite et les pires formes de travail des enfants

\footnotetext{
${ }^{31}$ Le seuil de pauvreté tel que mesuré par l'INS est constant en terme réel et relatif : il correspond au revenu des $10 \%$ les plus pauvres de la population en 1985 . Ce revenu était alors de 75.000 FCFA par tête et par an ; actualisé avec le taux d'inflation, le revenu correspondant au seuil de pauvreté est de $241.145 \mathrm{~F}$ CFA en 2008, soit approximativement 1,4 US\$ par tête et par jour.
} 
existant depuis 2007. Ce texte prévoit des sanctions pour tous les responsables directs des formes les plus graves d'exploitation des enfants y compris toute personne qui entretient des relations sexuelles avec un enfant (« consenties » ou pas). Enfin ce texte prévoit les mécanismes de prévention, de répression de la traite et du travail dangereux ainsi que la prise en charge des victimes. Par ailleurs la promulgation des textes n'est que le début, car il s'avère indispensable de garantir la disponibilité de suffisantes ressources humaines et financières pour leur application dans le quotidien.

Par ailleurs, quels problèmes posent le statut au travail des adolescentes commerçantes en rapport avec leurs conditions de travail et de vie? Et quel lien existe-il entre les conditions de travail et de vie des adolescentes commerçantes et certaines formes de déviances et marginalités?

\section{Objectifs et hypothèses}

L'objectif principal de cette étude est d'analyser les conditions de travail et de vie de ces petites commerçantes afin de savoir si celles-ci induisent des formes particulières d'exploitation. Pour atteindre cet objectif, l'hypothèse suivante a été formulée : Les conditions de travail et de vie difficiles des petites commerçantes dans le secteur informel favorisent chez elles le développement de comportements déviants ou marginaux (recherche de l'argent ou du gain facile) et l'accoutumance aux fléaux modernes (mendicité, prostitution, vol, délinquance, violence, etc.).

Deux théories ont été convoquées, l'une portant sur les théories victimologiques et l'autre sur les théories criminologiques du passage à l'acte. Les théories victimologiques consistaient (avant 1980) à attirer l'attention des chercheurs sur le rôle de la victime dans le passage à l'acte criminel. Mais audelà de cette période, les victimes ont été étudiées indépendamment du passage à l'acte et de la personnalité du délinquant. Les victimes doivent désormais être traités pour elles-mêmes, quelle que soit l'origine du malheur qui les frappe et du préjudice qu'elles subissent. D'où l'énonciation du concept de victimité (Mendelsohn, 1973), pour désigner la situation de toutes les victimes, dont les victimes d'actes délinquants ne forment qu'une variété, à coté des victimes de faits non fautifs, d'évènements fortuits ou de catastrophes naturelles. C'est à cette nouvelle approche que correspond ce que l'on appelle la criminologie victimologique ou encore «victimologie de l'action » (Fattah, 1981) pour laquelle la victimisation par actes délictueux est considérée comme une sorte de « risque social », au même titre que celle qui résulte d'autres évènements « Il ne s'agit plus de cristalliser les recherches sur la culpabilité de la victime comme dans la première victimologie, mais de porter un intérêt sur la reconnaissance et le traitement distincts des souffrances subies par les victimes : C'est-à-dire la seconde victimologie. Ces théories ont un rapport certain avec notre objet d'étude du moment où elles mentionnent l'existence 
d'« éléments communs permettant de découvrir les données générales qui rendent certains individus enclins à devenir des victimes à cause d'un potentiel réduit ou inexistant de résistance, du point de vue biopsychosocial » (Cario cité par Koudou, 2007).

Ainsi, l'adolescente au travail se présentera comme une victime de la réalité sociale ; mieux, "une victime totalement innocente » ou "victime inconsciente » ou encore «victime latente ou prédisposée (victime à prédisposition biopsychologique, socialement prédisposée, moralement ou psychologiquement prédisposée)» (Koudou, 2007). Elle sera aussi comptabilisée au rang des victimes biologiquement faibles au plan mental ou physique, socialement faibles, vulnérables. Certaines de ces théories victimologiques, plus pragmatiques et constructives, reposent également sur le vécu et l'expérience des victimes. Nous faisons allusion ici, à la théorie du style de vie quotidien des victimes ou théorie des opportunités de COHEN et FELSON (1979). Selon celle-ci, le passage à l'acte est exclusivement expliqué par rapport au mode de vie quotidien des victimes. Elles insistent sur les caractéristiques sociologiques, psychologiques et victimologiques des victimes. Ces théories nous permettront d'établir le lien entre style de vie (conditions de vie et de travail) et victimisation, à travers la vulnérabilité des petites commerçantes.

Notre travail pourrait aussi s'inscrire dans la perspective des théories des facteurs sociaux de la délinquance. Ici, Marx et Engels mettent l'accent sur les conditions économiques (théorie Marxiste- Léniniste); alors que CLIFFORD Shaw évoque les circonstances sociales et économiques d'une zone géographique déterminée (théorie écologique). La préoccupation de ces auteurs, c'est comment les conditions de travail et de vie ou les conditions économiques conduisent certains individus à être délinquants et d'autres non. Ici, ces théories pourront nous aider à appréhender les déviances et marginalités juvéniles des enfants en général, particulièrement celles des adolescentes au travail. Songeons également à la théorie de la dissocialité de Roger MUCCHIELLI (1965). Selon cet auteur, il faut partir de l'analyse du processus de socialisation pour comprendre la délinquance des jeunes. La société, note MUCCHIELLI, est une organisation avec des rôles différents et affectée par des drames tels la guerre, les chômages ; cette organisation oppose également une certaine résistance à l'intégration de tout sujet, cherchant à y entrer. Dans cette perspective, l'intégration d'une société est liée à l'acceptation active de celle-ci, à la perception exacte de celle-ci et à la prise en charge du rôle. L'échec du processus de socialisation peut conduire à la formation d'une personnalité dissociale (Koudou, 2007). Celle-ci comporte trois traits : la non acceptation de la société ; la perception sociale fausse de l'avenir et le rejet du rôle. L'échec du processus peut être lié aux conditions sociales difficilement maîtrisables, aux failles de la personnalité incapable de 
s'ajuster aux conditions de vie ou peu motivée de les assumer (Koudou, op.cit). Dans le cadre de notre objet, cette théorie nous permettra de ressortir le lien entre l'échec de socialisation des adolescentes (qui emmène les parents à réaliser que « la place de la jeune fille, ce n'est pas à l'école mais plutôt au travail ») et leur implication dans le monde du travail, notamment, celui du secteur informel du petit commerce.

\section{Méthodologie \\ Sites et participants}

Selon N'DA (Ndagijimana, 2013), la population est une collection d'individus (humains ou non) c'est-à-dire un ensemble d'unités élémentaires (une personne, un groupe, une ville, un pays) qui partagent des caractéristiques communes précises selon des critères définis. Les critères peuvent concerner par exemple l'étendue, l'âge, le sexe, la scolarité, le revenu etc.

Dans l'optique de mieux appréhender l'objet d'étude, la diversification des sources d'information fut nécessaire. A cet effet, la population d'étude est composée de toutes les catégories de la population d'Abidjan, et qui sont susceptibles de fournir des informations pouvant éclairer la compréhension de l'objet d'étude. Pour ce faire, nous avons enquêté sur la moitié de la ville, soit cinq communes (Abobo, Yopougon, Port-bouet, Adjamé, Treichville) choisies selon leur spécificité (populaire et populeux, résidentiel, administrative, etc.). Dans chacune de ces communes, nous identifions les endroits (rues, espaces) où il est susceptible de trouver des adolescentes qui exercent un commerce ambulants. Ainsi, les catégories sociales suivantes : Les adolescentes au travail dans le petit commerce, Parents, Tuteurs, familles d'accueil des enfants, chefs de communauté ethniques et religieuses, Employeurs ou les acteurs de lutte contre le travail des enfants (BIT, BICE, UNICEF, OIT, AIDF....).

Les adolescentes au travail, les parents ou familles d'accueil sont déterminants dans la constitution de notre population d'enquête, dans la mesure où, ils sont les acteurs principaux acteurs par conséquent, leur avis est incontournable dans cette étude. Les interroger contribuera à comprendre l'environnement dans lequel, vivent les enfants et les difficultés qu'ils rencontrent sur leurs lieux de travail. Quant aux Employeurs, nous les avons inclus dans la population d'enquête, afin d'avoir certaines informations sur la nature de leur relation avec les enfants qu'ils emploient.

Par ailleurs, l'utilité de la prise en compte des responsables des services administratifs (les acteurs de lutte) et les chefs de communauté ethniques et religieuses dans l'élaboration de la population d'enquête, réside dans le fait que ceux-ci sont le plus souvent interpellés sur la question des conditions de travail et de vie des enfants.

En définitive l'échantillon des adolescentes enquêtées par outils est au nombre de 150 soit 30 par commune et les employeurs 50. Pour donner un 
caractère extrapolable aux résultats de l'enquête, nous avons soumis la sélection des sujets de l'échantillon aux techniques de sondages empiriques. Comme la règle l'indique, la technique des itinéraires a été associée à la technique aréolaire pour la conduite de l'enquête sur le terrain. Ces deux (2) méthodes s'illustrent comme les procédés les mieux adaptés à l'étude. Elles ne permettent donc pas de produire des marges d'erreurs ; le but ici étant de se « rapprocher au maximum » d'un tirage rigoureusement aléatoire et d'une équiprobabilité des chances, Spécialistes de la question de l'enfance "psychologues, sociologues, éducateurs spécialisés, criminologues", Elus locaux, Responsables des institutions et Organisations Non Gouvernementales de protection et d'aide à l'enfance en difficulté 30, Forces de Défense et de Sécurité , Population civile 50 car vue notre objet d'étude, nous avons été orienté vers certains responsables de ces institutions.

\section{Méthodes}

Dans le cadre de l'élaboration de notre étude, trois (03) méthodes de recherche ont été utilisées : la méthode systémique, la méthode dialectique et la méthode ethnographique. La première, a permis de considérer le cadre familial comme un système social dont l'on cherchera à comprendre à travers les mécanismes sociaux qui conduisent les enfants au travail. Dans la méthode dialectique, nous avons mis l'accent dans l'explication des contradictions entre la réalité dans les familles et l'insertion dans une activité économique dont les enfants font l'objet. La troisième méthode met l'accent sur la culture. Culture pouvant justifier, le choix du travail des enfants. Nous avons utilisé cette méthode dans le but de comprendre la présence des enfants dans le petit commerce.

Sur le plan du traitement des données, nous avons utilisé à la fois l'analyse quantitative et l'analyse qualitative. Dans le premier cas, nous avons mis l'accent sur le traitement statistique des données afin de mesurer l'ampleur du phénomène du travail des enfants dans les statistiques des structures enquêtées, les différentes formes qu'elle prend et son rapport avec les familles ; dans le second cas, le vécu des sujets a été utilisé en vue d'approfondir les logiques d'adaptation des enfants victimes d'exploitation et les réactions des employeurs, sans oublier la nature et l'état des dispositifs de prise en charge déployés par les acteurs de terrains. Cette analyse nous a permis de suggérer des relations entre des variables (croyances socioculturelles et économiques, exploitation des enfants, secteur informel).

Aussi, nous avons utilisé un questionnaire, un guide d'entretien qui ont été administrés individuellement et collectivement dans le cadre des focus groupes.

De manière précise, l'étude phénoménologique a permis de saisir les pratiques, perceptions, opinions, attitudes, significations et représentations 
liées au vécu de ces adolescentes au travail dans le secteur informel du petit commerce en milieu urbain.

La mise en œuvre des différents outils de recueil et d'analyse des données ont favorisé une présentation quantifiés des résultats sous forme de tableaux et de graphiques avec l'aide du logiciel SPSS et le tableur d'Excel.

\section{Résultats}

La présentation des résultats s'articuleront autour des conditions de travail (I) et des conditions de vie (II).

\section{I- CONDITIONS DE TRAVAIL DES ADOLESCENTES COMMERCANTES}

\section{I-1. Les situations de travail dangereux chez les adolescentes commerçantes}

\section{I-1-1. La durée de travail}

Les adolescentes commerçantes interrogées travaillent en moyenne six (6) jours par semaine et dix (10) heures par journée de travail. Le volume horaire moyen de travail par semaine et par fillette est de soixante (60) heures. La moyenne horaire de travail des adolescentes commerçantes reste élevée et supérieure à quarante huit (48) heures en moyenne par semaine quel que soit le statut au travail de ces adolescentes.

Tableau $\mathrm{n}^{\circ} \mathbf{1}$ : Charge horaire hebdomadaire de travail des petites commerçantes dans le secteur informel (\%).

\begin{tabular}{|c|c|c|c|c|c|c|}
\cline { 2 - 6 } \multicolumn{1}{c|}{} & \multicolumn{2}{c|}{ Secteur d'activité } & \multicolumn{4}{c|}{ Statut au travail } \\
\cline { 2 - 7 } \multicolumn{1}{c|}{} & Commerce & Services & $\begin{array}{c}\text { Aide } \\
\text { familial }\end{array}$ & Apprentie & $\begin{array}{c}\text { Travail } \\
\text { rémunéré }\end{array}$ & $\begin{array}{c}\text { Travail } \\
\text { indépendant }\end{array}$ \\
\hline $\begin{array}{c}\text { Ensemble des } \\
\text { petites } \\
\text { commerçantes }\end{array}$ & 57,2 & 62,4 & 63,1 & 66,1 & 61,8 & 50,7 \\
\hline $\begin{array}{c}\text { Adolescentes } \\
\text { victimes } \\
\text { d'exploitation }\end{array}$ & 70,6 & 87,5 & - & - & 71,9 & - \\
\hline
\end{tabular}

\section{Source : Enquête du terrain}

L'analyse des résultats de l'enquête révèle que $85,7 \%$ des adolescentes commerçantes interrogées travaillent pendant plus de quarante (40) heures par semaine. A l'observation faite sur le terrain, on constate que plus de huit adolescentes au travail dans le secteur informel du petit commerce sur dix est soumise à une charge de travail trop importante.

L'observation des données du tableau ci-dessus (moyenne horaire de travail des petites commerçantes et pourcentage travaillant pendant plus de quarante heures par semaine) permet de faire les commentaires suivants :

- La charge horaire de travail est plus élevée chez les adolescentes ayant fait l'objet de traite quels que soit le secteur d'activité, que ce soit sur la base de la moyenne horaire de travail des fillettes par semaine ou par 
taux d'adolescentes travaillant pendant plus de 40 heures par semaine.

- Les adolescentes commerçantes sont généralement soumises à des conditions horaires de travail plus rudes, quels que soit l'indicateur retenu (moyenne horaire de travail par semaine ou taux de fillettes travaillant pendant plus de 40 heures par semaine).

- Les adolescentes commerçantes non indépendantes (adolescentes travaillant sous la tutelle d'une personne adulte) sont astreintes à des horaires de travail plus rudes que leurs camarades indépendantes (qui ont l'avantage de déterminer elles-mêmes leurs horaires de travail) quel que soit l'indicateur retenu.

Par rapport au rythme de travail, une jeune commerçante à Adjamé parle de celui-ci en ces termes : " Chez ma patronne, tout le monde se réveille à cinq (5) heures trente (30) minutes. On est au marché avant le lever du soleil, et on ne rentre à la maison qu'à partir de dix-huit (18) heures ». En effet, de manière générale le commerce auquel s'adonnent la majorité de ces adolescentes, dure toute la journée. Il commence autour de sept (07) heures, et prend fin vers dix-sept (17) heures voire dix-huit (18) heures. Aussi, la tâche de ces adolescentes, rappelons-le, ne se limite pas seulement au commerce exercé dans les rues. En effet, elles s'occupent aussi des travaux ménagers, de sorte que le travail commence à la maison, se poursuit dans la rue pour s'achever à la maison. Un tel programme, à l'évidence, laisse très peu de place au repos. Voici à propos, les temps forts de la journée de travail de Kady : « j’habite Adjamé. Je marche pour arriver à Cocody où je vends mes oranges. Le matin, je me lève à $5 \mathrm{~h} 00$ pour faire les travaux de la maison. A $7 \mathrm{~h}$, je sors avec ma marchandise et je commence à vendre. J'arrête à $17 \mathrm{~h} 30$ et je rentre à la maison ».

Cette journée de travail de Kady ne diffère pas de celle des autres filles de son âge. Pire, il faut mentionner le cas de celles qui habitent loin comme Fatou qui vient de Yopougon. C'est généralement à pieds ou en gbaka $^{32}$, qu'elle rejoint son lieu de travail. Pour le retour, c'est toujours les mêmes moyens de locomotion qu'elle utilise. Elle «s'amuse » souvent à frauder le bus pour regagner la maison. La journée de travail est en général encore plus longue que celle qui vient d'être décrite quand on y ajoute les travaux ménagers avant le départ et le retour à la maison. En fait, la plupart de ces filles, dont la maturation physique, psychologique et psychique n'est pas encore achevée, est sur pied de 5 h 00 à 20 h 30 et plus; soit une journée de quinze (15) heures au minimum, dans des conditions pénibles (position debout pour vendre dans la rue et position courbée pour faire le ménage à la maison).

A cela, nous avons également constaté que la charge de travail des employeurs ne tient pas compte de l'âge de ces adolescentes mais plutôt des

${ }^{32}$ Minicar de transport en commun. 
impératifs liés à la recherche de la productivité et de la rentabilité. Les adolescentes commerçantes se voient ainsi imposer une charge horaire de travail presque égale à celle des travailleurs adultes de plus de 17 ans. Selon les employeurs interrogés, les adolescentes travaillent en moyenne pendant 10,3 heures par journée de travail contre 10,4 heures pour les adultes de 18 ans et plus. Au vu de cette situation, nous sommes arrivés à la conclusion que les adolescentes au travail dans le secteur informel du petit commerce travaillent autant et souvent même plus que les adultes.

\section{I-1-2. Le travail dans des environnements à risques pour les adolescentes}

Certaines adolescentes au travail dans le secteur informel du petit commerce sont exposées à l'effet de produits dangereux ${ }^{33}$ par manipulation directe ou par exposition indirecte sur les lieux de travail. L'exposition directe ou indirecte à l'effet de produits dangereux concerne $27,3 \%$ des petites commerçantes interrogées. Cette exposition aux produits dangereux concerne davantage les adolescentes du secteur commerce et précisément $27,3 \%$ de celles qui exercent les petits services informels. La charge horaire de travail est à la fois déterminée par le secteur d'activité et les caractéristiques des adolescentes qui y exercent. Par contre, les risques liés aux conditions physiques de travail semblent uniquement déterminés par le secteur d'activité des adolescentes. En effet, la probabilité pour une petite commerçante de manipuler les outils ou produits dangereux dépend uniquement des tâches qu'elle est amenée à exécuter dans le cadre de son travail (donc du secteur dans lequel elle travaille).

En plus de la pénibilité des tâches, les adolescentes s'exposent aux divers dangers spécifiques de la rue. En effet, certaines petites commerçantes affirment avoir été victimes d'agressions physiques. Adja raconte ainsi sa mésaventure : « un jour, il y a deux (2) jeunes (voyous) qui m'ont agressée (physiquement) au marché. Ils m'ont frappé et voler mon argent ». Compte tenu de leur âge, grand nombre de ces adolescentes est la cible privilégiée des bandes de jeunes (voyous) : « souvent, ils boivent nos sachets d'eau, sans payer. Et quand tu parles, ils te frappent», ajoute D. K. (12 ans), vendeuse d'eau glacée.

Face à toutes ces agressions, les adolescentes, encore trop jeunes, restent sans défense. En effet, la plupart des adolescentes dans le petit

\footnotetext{
${ }^{33}$ La notion de produits dangereux désigne les substances dont la manipulation est susceptible de présenter des dangers pour la santé et le bien-être des petites commerçantes soit parce qu'elles sont directement nocives soit parce qu'elles sont capables de produire des effets néfastes à plus ou moins long termes sur la santé de ces adolescentes qui les manipulent ou qui y sont exposées. Dans le cadre de cette étude, trois produits ont été identifiés comme dangereux. Ce sont : le vernis (colle, diluant, peinture), les acides et les autres produits dérivés du pétrole.
} 
commerce n'ont pas de protection ni d'expérience. Par conséquent, elles sont contraintes de subir les mauvaises conditions de travail, des vols, bref des situations où elles sont impuissantes : "Quand les deux (2) jeunes (voyous) m'ont frappé et m'ont arraché mon argent, personne n'est venue à mon secours. Je n'ai pas pu me défendre parce que je suis trop petite ", raconte Adja. En ce sens, le travail est subi et il affecte la construction de l'image positive et l'estime que les adolescentes ont d'elles-mêmes.

Une autre nuisance pour les adolescentes au travail dans le secteur informel du petit commerce, provient de la pollution atmosphérique causée par le vieillissement du parc automobile et de la congestion des voies de circulation. Elle constitue le premier enjeu environnemental, loin devant l'effet de serre et le bruit. En effet, les moyens de transports émettent des polluants qui ont un impact certain sur la santé des petites commerçantes. Il s'agit de l'oxyde d'azote ( $\mathrm{NOx})$, le monoxyde de carbone $\left(\mathrm{CO}_{2}\right)$, d'hydrocarbures volatils $(\mathrm{HC})$, de dioxyde de soufre $\left(\mathrm{SO}_{2}\right)$ et du plomb $(\mathrm{Pb})$. Comme conséquence, le taux de concentration de $\mathrm{CO}_{2}$ dans l'air à Abidjan est dix fois supérieur à celui des autres villes de la Côte d'Ivoire. L'impact de ces polluants sur la santé des petites commerçantes est indéniable. En l'absence de statistiques fiables, on peut cependant noter une forte augmentation de différentes maladies causées par la pollution de l'air, dont les maladies respiratoires, les allergies, les maladies de la peau, les maladies neurologiques, les infarctus du myocarde, les céphalées et vertiges, les cancers du poumon et de la vessie, le saturnisme (infection par le plomb), etc. Les dysfonctionnements du système des transports urbain à Abidjan constituent par conséquent un problème économique mais aussi un problème de santé publique. Mais le danger le plus immédiat qui guette en permanence les adolescentes dans la rue, demeure à n'en point douter, les accidents de voitures. Et la majorité d'entre elles, en sont conscientes.

Pour elles, les conditions de vie et de travail, aussi pénibles que dangereuses soient-elles, ne paraissent pas constituer une préoccupation. Certes, certaines d'entre elles racontent leurs mésaventures, mais la plupart les minimisent au point de ne même pas mentionner les mauvais traitements qu'elles subissent parfois dans le cadre de leur activité. Et pourtant, il suffit d'observer attentivement les conditions dans lesquelles ces adolescentes travaillent pour s'en rendre compte. Seule l'innocence propre à leur âge, amène ces fillettes à accepter ces travaux contraignants et harassants. 
I-2. Un travail « sans salaire »

I-2-1. Un revenu mensuel moyen inférieur au SMIG

Tableau $\mathbf{n}^{\circ} \mathbf{2}$ : Revenu mensuel moyen des petites commerçantes (en francs CFA).

\begin{tabular}{|c|c|c|c|c|c|c|c|}
\hline & \multicolumn{4}{|c|}{ Statut au travail } & \multicolumn{3}{c|}{ Mode d'hébergement } \\
\cline { 2 - 7 } & $\begin{array}{c}\text { Aide } \\
\text { familiale }\end{array}$ & $\begin{array}{c}\text { Apprenti } \\
e\end{array}$ & $\begin{array}{c}\text { Travail } \\
\text { rémunér } \\
\text { é }\end{array}$ & $\begin{array}{c}\text { Travail } \\
\text { indépenda } \\
\text { nt }\end{array}$ & $\begin{array}{c}\text { Vivant } \\
\text { chez un } \\
\text { parent }\end{array}$ & $\begin{array}{c}\text { Vivant } \\
\text { chez un } \\
\text { tuteur }\end{array}$ & $\begin{array}{c}\text { Vivant chez } \\
\text { un } \\
\text { employeur }\end{array}$ \\
\hline $\begin{array}{c}\text { Adolescente } \\
\text { sommerçan } \\
\text { tes }\end{array}$ & - & 6500 & 12000 & - & - & 12000 & 11222 \\
\hline
\end{tabular}

Source : Enquête de terrain

La charge horaire de travail des employeurs, comme nous l'avons signifié plus haut, ne tient pas compte de l'âge des petites commerçantes mais plutôt lié à la recherche de la productivité et de la rentabilité. Paradoxalement, les adolescentes perçoivent une rémunération bien inférieure au vu de leurs rendements.

L'observation des revenus mensuels des adolescentes commerçantes donne lieu à plusieurs commentaires. D'abord, le revenu des fillettes est inferieur au SMIG en Côte d'Ivoire qui est de $36607 \mathrm{~F} \mathrm{CFA}^{\mathbf{3 4}}$ environ par mois. Ensuite, les petites commerçantes non indépendantes perçoivent une rémunération faible que le revenu net de travailleuses indépendantes. Pire, ces adolescentes perçoivent des rémunérations faibles, alors qu'elles ont des charges horaires très élevées. Enfin, celles qui vivent chez leurs employeurs, perçoivent une rémunération moyenne plus faible que celles qui vivent chez leurs parents où avec un tuteur non employeur. Aussi, notons nous qu'en dépit du rythme intense et de la dureté des travaux auxquels sont soumises ces adolescentes contractuelles, il nous est revenu que de nombreuses exploitantes, ne respectaient pas les clauses du contrat. A ce propos, nous avons reçu un témoignage éloquent d'un responsable d'ONG au Plateau : « les jeunes filles qui ont des salaires, n'ont pas de repos. Nous connaissons le cas d'une petite fille, qui a vendu durant plusieurs années pour sa patronne sans recevoir un seul centime de ce qu'on lui avait promis. Parfois, les patronnes se basent sur des prétextes ou des accusations non fondées pour ne pas payer ces jeunes filles. Elles accusent ces adolescentes de vol, de paresseuse, et pire de coucher ou de vouloir coucher avec leurs maris, pour ne pas les payer $"$.

Un autre type de raison qui rend compte du non-respect de ses engagements par l'employeur, apparaît dans le discours ci-après. «Pour ne pas payer ces jeunes filles, les employeuses avancent souvent qu'elles ont eu à faire trop de dépenses par rapport à ces dernières notamment en

${ }^{34}$ Code du travail (2000), Edition juridique de Côte d'Ivoire. 
matière de santé, d'habillement » (Propos recueillis auprès d'un responsable de l'UNICEF). Comme on le constate, à travers ces discours, de nombreux prétextes sont avancés, ou du moins des stratégies sont déployées, par des employeurs pour ne pas respecter les clauses du contrat qui les lient à ces adolescentes. Devant les conditions de travail difficiles et le non-respect des engagements par les employeurs, de nombreuses adolescentes sont naturellement mobiles dans l'espace et dans le temps.

\section{I-2-2. Des recettes conséquentes, versées à la tutrice ou à l'employeur.}

Le petit commerce exercé par les adolescentes dans les rues de la ville d'Abidjan fait gagner de l'argent tous les jours. La recette varie en général entre mille cinq cents (1 500) et deux mille cinq cents (2 500) voire trois mille (3 000) francs CFA. Cette recette journalière, à première vue, semble importante si l'on tient compte de l'âge de ces filles et de la quantité de marchandises qu'elles vendent au cours de la journée. C'est donc au su des profits qu'ils peuvent retirer du travail de ces adolescentes, que les parentstuteurs et les employeurs choisissent la destination de la capitale économique ; et cela dans l'espoir de voir leurs filles gagner rapidement de l'argent.

Aussi, contrairement au travail exercé par les petites domestiques, un fait important est à noter. En effet, dans le cadre du petit commerce exercé par les adolescentes, les tutrices ou les employeurs n'exigent souvent pas de ces dernières aucun montant pour la recette journalière. Mais en réalité, cette attitude paradoxale a une explication. Ne pas demander une recette journalière à ces adolescentes, peut être perçu comme une preuve de laxisme, de désintéressement ou tout simplement la manifestation d'une certaine affection à l'égard de ces adolescentes du fait des liens de parenté. Toutefois, l'employeur met en place un mécanisme subtil de contrôle que la naïveté des filles ne permet pas d'appréhender tout de suite. A ce titre, voici les propos de Korotoum lors de l'une de nos enquêtes : « ma tante compte les sachets d'eau avant de me donner. Elle me dit aussi qu'elle ne veut pas de manquant; donc de m'arranger pour que l'argent soit juste ». A y regarder de plus près, on note qu'il y a non seulement une obligation de recette (même si à priori cela n'est pas visible) mais également l'exactitude de celleci. Celles-ci portent sur la bonne gestion de la quantité des marchandises reçues et aussi sur la recette. En effet, en cas de manquant ou de mauvaise recette, les adolescentes se le voient reprocher, le plus souvent de manière violente. C'est ce que révèle Korotoum en ces termes : « le jour où çà ne marche pas bien ou bien le jour où le nombre de sachets d'eau ne correspond pas à l'argent que je lui envoie, ma tante me frappe ».

De même qu'aucun montant n'est fixé par rapport à la recette, de même on constate que le travail des adolescentes n'est pas rémunéré en tant que tel. Elles ne sont payées ni pour le commerce qu'elles exercent ni pour les 
travaux domestiques qu'elles exécutent à la maison. Mais est-ce à dire que les adolescentes travaillent sans aucune contrepartie financière ? Assurément pas. En effet, le salaire de ces adolescentes se paye autrement qu'en numéraires (caractéristique des rapports contractuels liant un employé à son employeur). Dans la situation d'espèce, il se paye en nature, sous forme de cadeaux, notamment des vêtements. Quand bien même elles ne sont pas nombreuses, certaines petites commerçantes arrivent à épargner. Ici, elles gardent une partie de leur salaire (pour celles qui sont payées) ou de la recette tirée des pourboires ou d'autres activités parallèles (pour celles qui ne sont pas liées par un contrat formel de travail) dans une caisse (sorte de tirelire) ou l'investissent dans la tontine. Quant à l'autre partie, elle est reversée à la tante ou à l'employeur. En effet, certaines filles déposent leur épargne dans une caisse qu'elles confient à une tierce personne. C'est cette dernière qui gère les avoirs des adolescentes en les répartissant en achats d'habits de fête et en épargne pour le retour dans la famille biologique. La gestion de leurs revenus est en général laissée en toute confiance à la tutrice qui en a l'entière responsabilité. Cette dernière, en retour, n'exige à son employée aucune contribution particulière aux dépenses de la maison (frais de logement et de nourriture).

Ceci montre bien qu'il s'agit d'une situation inscrite dans une stratégie familiale dans laquelle chacun joue sa partition. Car, accueillir à Abidjan la fille d'un parent, fut-il proche ou éloigné, pour lui ouvrir des opportunités de se faire de l'argent, est conçu et perçu comme une des formes d'expression de la solidarité qui caractérise les familles nombreuses aux prises avec les difficultés économiques. Et même, face à certaines urgences, une somme est prélevée sur l'épargne ou l'économie de ces adolescentes pour être envoyée aux parents de ces dernières. Toutefois, il convient de souligner dans cette ambiance, que certaines adolescentes (17 et 18 ans) disposent, d'une véritable autonomie dans l'exercice de leur activité. Mais en général, en dépit de cette autonomie, la gestion de leurs économies demeure la responsabilité de la tante.

Comme on le voit, ces adolescentes se destinent, en général, volontiers au petit commerce et semblent s'y plaire à cause de sa facilité car il ne nécessite pas d'apprentissage particulier ni d'investissement à gros budget. Mais en réalité, l'activité de petit commerce dans les rues a ses exigences : une endurance physique pour parcourir les rues et artères des communes, et ce, à voix forte, car les filles doivent crier pour racoler les clients. Même si elles ne s'en aperçoivent pas toutes, le petit commerce exercé dans le secteur informel nécessite un minimum d'intelligence. Car les adolescentes doivent faire montre d'esprit d'initiative et d'ingéniosité pour rentabiliser leur journée.

En définitive, la majorité des adolescentes exerçant dans le secteur informel du petit commerce dans le district d'Abidjan, ne sont pas rémunérées en tant que tel selon un contrat écrit ou verbal. Au regard des conditions de 
travail des petites commerçantes dans le secteur informel, on peut retenir que celles-ci paraissent être particulièrement plus contraignantes, quand il s'agit de contrats formels ou tacites, et mettent sous pression des adolescentes sans défense face à des adultes qu'elles ne connaissent pas ; ce qui ne semble pas être toujours le cas de celles censés travailler avec leurs « parents ».

\section{II- CONDITIONS DE VIE DES ADOLESCENTES COMMERCANTES II-1. Conditions de logement}

Tableau $\mathbf{n}^{\circ} \mathbf{3}$ : Conditions de logement des petites commerçantes.

\begin{tabular}{|c|c|c|c|c|c|c|}
\hline & \multicolumn{2}{|c|}{ Tranche d'âge } & \multirow{2}{*}{$\begin{array}{l}\text { Fillettes ayant } \\
\text { effectué une } \\
\text { migration }\end{array}$} & \multirow{2}{*}{$\begin{array}{l}\text { Fillettes } \\
\text { ayant fait } \\
\text { l'objet de } \\
\text { traite }\end{array}$} & \multirow{2}{*}{$\begin{array}{l}\text { Ensemble de } \\
\text { l'échantillon } \\
\qquad(N)\end{array}$} & \multirow{2}{*}{$\%$} \\
\hline & $11-14$ & $15-18$ & & & & \\
\hline Avec parents & 26 & 14 & 05 & 00 & 45 & 30 \\
\hline $\begin{array}{c}\text { Avec } \\
\text { employeurs }\end{array}$ & 17 & 19 & 16 & 08 & 60 & 40 \\
\hline Seules & 00 & 00 & 00 & 00 & 00 & 00 \\
\hline Avec tuteurs & 09 & 12 & 12 & 03 & 36 & 24 \\
\hline Avec amis (es) & 00 & 01 & 05 & 03 & 09 & 06 \\
\hline Total $(N)$ & 52 & 46 & 38 & 14 & 150 & 100 \\
\hline
\end{tabular}

Source : Enquête de terrain

Moins de la moitié des adolescentes commerçantes interrogées (30\%) ne vivent avec aucun de leurs parents biologiques. La majorité d'entre elles vit avec leur employeur (40\%) ou leur tuteur non employeur (24\%). La situation des fillettes hébergées par leur employeur est particulièrement préoccupante. En effet, parce qu'elles sont particulièrement prises en charge, les adolescentes de 10-14 ans, plus enclins à migrer à la recherche d'un emploi rémunéré, sont plus nombreuses à vivre chez leurs employeurs et à être ainsi exposées au risque d'une exploitation exagérée et abusive de leur force de travail. Celles ayant fait l'objet de traite vers Abidjan (14 concernées sur les 150 interrogées) tout comme celles qui ont effectué une migration $(n=38)$ ont tendance à vivre chez leurs employeurs. En général, ces filles habitent dans des logements exigus, ne dépassant guère en moyenne deux (2) pièces c'est-à dire un séjour et une chambre. Dans la quasi-totalité des cas, les adolescentes rencontrées dorment au salon ${ }^{35}$. "On est beaucoup à la maison alors que c'est petit. Nous, les enfants, on dort au salon » dit Karidja.

De plus, les conditions dans lesquelles dorment les adolescentes la nuit, sont marquées par la promiscuité. De nombreux enfants qu'elles étaient chez leurs parents biologiques, elles se retrouvent dans des ménages surchargés chez leurs tutrices ou employeurs à Abidjan. Tout le monde, en dehors des

${ }^{35}$ Espace communément partagé avec d'autres enfants ou même des adultes ; la chambre étant réservée au maître ou à la maîtresse de la maison. 
employeurs, dort au salon; et ce, sans distinction de sexe ni d'âge. Certes les adolescentes n'ont signalé aucun acte répréhensible, mais il convient de souligner qu'une telle situation peut comporter des risques et / ou des tentations. Ces situations désavantageuses ne sont pas seulement apparentes puisque ces logements sont pour la plupart situés dans les quartiers précaires ou périphériques de la capitale économique. Et comme conséquence, ces logements sont mal raccordés aux réseaux d'eau et d'électricité et n'offrent pas assez de confort de par leur proximité, à l'infrastructure économique de base. Qu'en est-il de l'accès au réseau d'eau potable et d'assainissement ?

L'examen du mode d'approvisionnement en eau potable dans ces quartiers précaires montre que l'accès à l'eau potable de réseau ou de fontaine publique reste très limité. Quant à l'assainissement, l'analyse du mode d'évacuation des eaux usées montre que ces ménages évacuent leurs eaux usées dans la nature ; le raccordement au réseau d'évacuation des eaux usées dans les quartiers précaires n'existant pas. Il en est de même pour l'accès à l'électricité et aux moyens de communication. Il ressort de tout ce qui précède, qu'il existe de fortes disparités entres les ménages dits aisés et les ménages pauvres en matière de conditions d'habitation. C'est ainsi que les faibles taux de raccordement au réseau d'eau potable, d'assainissement liquide, d'électricité, etc. sont enregistrés parmi les ménages où il existe des adolescentes au travail. Il en résulte que les adolescentes au travail dans le secteur informel du petit commerce issues en majorité de ces quartiers précaires, vivent à n'en point douter, dans des conditions de précarité, de promiscuité, d'insalubrité, et de pauvreté économique.

\section{II-2. Relations avec le milieu familial}

Cette relation se présente comme une organisation verticale facteur de perturbation pour les adolescentes.

\section{II-2-1. Rejet et fuite de la famille.}

Tableau n ${ }^{\circ}$ : Contact des petites commerçantes avec leurs familles biologiques.

\begin{tabular}{|c|c|c|c|c|c|c|}
\hline \multicolumn{1}{c|}{} & \multicolumn{2}{|c|}{ Tranche d'age } & $\begin{array}{c}\text { Enfants } \\
\text { migrants }\end{array}$ & $\begin{array}{c}\text { Enfants victime de } \\
\text { traite }\end{array}$ & $\begin{array}{c}\text { Effectif } \\
(\mathbf{N})\end{array}$ & $\%$ \\
\cline { 2 - 7 } $\begin{array}{c}\text { Garde des } \\
\text { contacts avec la } \\
\text { famille }\end{array}$ & 28 & 33 & 25 & 10 & 96 & 64 \\
\hline $\begin{array}{c}\text { Plus aucun } \\
\text { contact avec la } \\
\text { famille }\end{array}$ & 14 & 05 & 07 & 28 & 54 & 36 \\
\hline Total & 42 & 38 & 32 & 38 & $\mathbf{1 5 0}$ & $\mathbf{1 0 0}$ \\
\hline
\end{tabular}

Source : Enquête de terrain

Certaines adolescentes au travail dans le secteur informel vivent dans une situation d'isolement vis-à vis de leurs familles d'origine. L'enquête a en 
effet révélé que 36\% des fillettes n'ont plus le moindre contact (même par courrier ou échanges téléphoniques) avec leur famille. Si en général, le taux de adolescentes commerçantes vivant dans cette situation d'isolement affectif reste faible, on note tout de même que le phénomène prend des proportions inquiétantes dès lors qu'il s'agit d'enfants migrants ou ayant fait l'objet d'une traite. Cela nous conduit à affirmer que trois (03) adolescentes au travail dans le secteur informel du petit commerce sur dix (10) n'ont plus aucun contact avec leur famille.

Les problèmes des adolescentes commerçantes liés aux conflits récurrents entre leurs employeurs ou « parents » (cris, insultes, coups, etc.) et la subordination dont elles sont l'objet à cause du système dans lequel elles vivent et travaillent font que le foyer familial devient inévitablement pour elles un lieu de mal-être. Bon nombre d'adolescentes fuient alors l'institution familiale de façon progressive ou brusque suite à cette violence intra-familiale et aux conditions de travail et de vie parfois extrêmes. Ces enfants et adolescentes vivent ensuite dans la rue, souvent en bandes, et trouvent rapidement, en plus de la délinquance et du trafic de drogues, l'exploitation et la violence sexuelle commise par des clients et/ou des proxénètes en quête de main d'œuvre mineure dans les maisons closes. Les nombreux témoignages d'adolescentes prostituées recueillis attestent ce phénomène de vulnérabilité et expriment cette distribution inégale de pouvoir entre les générations.

Le rejet des adolescentes du cadre familial induit d'autre part l'irresponsabilité des parents ou des employeurs. En réalité ceux-ci ne font rien pour ramener ces adolescentes à la maison. Tout ce passe comme s'ils consentaient à la fuite de celles-ci, se débarrassant du coup de tout scrupule. En effet, les menaces, la précarité et les éventuels dangers dont elles sont permanemment l'objet n'entament en rien la volonté « machiavélique » de ces parents ou employeurs. Interrogée la petite Barakissa nous avouera qu' « elle est parti de la maison familiale depuis deux ans ». Elle ajoutera ceci : « ma tutrice m'a frappée, après elle m'a dit de ne pas dormir dans sa maison et puis moi je suis parti, je ne suis plus revenue et personne à la maison n'est venue me chercher » Et pourtant il est avéré que la participation de ces adolescentes aux charges familiales est considérable, à tout le moins utile. Il s'en suit que les parents ou employeurs, prétextant fallacieusement d'une erreur ou maladresse de l'adolescente l'excommunie, la bannit de sorte à ne pas lui reverser son dû ou son salaire

\section{II-2-2. La violence intra-familiale source de désorganisation de la cellule}

Les conditions de vie dans lesquelles sont amenées à vivre les petites commerçantes rendent leur survie précaire et les privent d'un niveau de vie décent du point de vue matériel. Les relations au sein de la famille se dégradent petit à petit, parfois jusqu'à la séparation. Et la pauvreté seule n'est pas une 
raison suffisante des mauvaises conditions de vie des adolescentes. Le grand problème est tout ce qui en découle et dont les petites commerçantes sont victimes, notamment les diverses maltraitances physiques et psychologiques. La plupart des petites commerçantes rencontrées vivent dans des familles recomposées, où le nouveau conjoint est parfois source de tension. Des fois, il arrive que ce dernier maltraite et rejette les enfants qui ne sont pas de lui. Lorsque c'est une belle-mère qui pose problème, une maltraitance psychologique s'ajoute fréquemment à la maltraitance physique, ou bien il peut y avoir une maltraitance uniquement psychologique, mais qui n'est pas moins douloureuse. Souvent la petite commerçante est considérée comme un poids, une bouche de plus à nourrir... Et quand l'argent fait défaut dans le foyer, c'est la petite fille qui, en premier, est perçue comme une charge. L'alcoolisme du père et les mauvais traitements qu'il inflige à toute la famille est un autre motif fréquent de la désorganisation de la cellule familiale dans laquelle vivent ces adolescentes. Mieux, il est à la base de l'éclatement de celle-ci, puis de la séparation des adultes. Dans une telle situation, les traitements à l'égard des enfants diffèrent: l'adolescente ne peut plus recevoir assez de nourriture, s'habiller décemment, avoir droit aux divertissements, etc. car « il faut faire un choix ».

Nous pouvons donc conclure que la médiocrité des conditions économiques n'est pas la seule responsable des conditions de vie des adolescentes. Les intérêts personnels des uns et des autres entrent en jeu pour déterminer qui, dans la famille, bénéficiera de meilleures faveurs. Malheureusement pour les adolescentes, les marâtres et parâtres, dès leur entrée dans la famille, écartent souvent les autres enfants pour favoriser l'éducation des leurs.

\section{II-2-3. La vie familiale : un isolement dans la promiscuité, la précarité et la pauvreté.}

Notre champ d'analyse sera focalisé uniquement sur les ménages. Le choix des ménages comme champ d'analyse au détriment des adolescentes objet de notre étude, est dicté par le fait que ces jeunes filles ne sont pas maîtresses de leur sort mais plutôt de leur entourage familial et des conditions d'habitation et de vie de leurs ménages. En effet, les conditions d'habitation des ménages (les caractéristiques relatives au logement) sont susceptibles d'avoir des effets sur l'état de santé, de condition d'être et de cadre de vie des membres du ménage (appauvrissement en eau potable, disposition de l'électricité et des éléments de confort de toilettes, bains, etc.). Elles permettent également d'avoir une idée sur le niveau socioéconomique des ménages ; le but étant de montrer que les cadres de vie les plus modestes, sont associés au travail des enfants. Ainsi, l'enquête a montré que la plupart des ménages en milieu urbain ayant des jeunes enfants travailleurs n'est pas propriétaires de 
leurs logements. Ces ménages sont en général locataires de leurs logements d'habitation. La location (statut de ces logements) reste importante en milieu urbain en s'adjugeant l'un des premiers rangs du point de vue importance.

Quant au taux d'occupation de ces logements, il s'analysera en termes de nombre de pièces. En effet, l'analyse du nombre de pièces selon le milieu de résidence, montre que les ménages urbains ayant des adolescentes au travail, vivent dans des logements moins spacieux voire exigus par rapport à leurs homologues des milieux ruraux. En dehors de ceux ou celles chez qui elles vivent, les relations familiales des petites commerçantes à Abidjan se limitent aux parents qui les ont amenées et chez qui elles habitent.

\section{II-3. Situation nutritionnelle et sanitaire}

Tableau $n^{\circ} 5$ : Recours sanitaires des adolescentes en cas de pathologie.

\begin{tabular}{|c|c|c|c|c|c|c|}
\hline & \multicolumn{2}{|c|}{ Tranche d'âge } & \multirow{2}{*}{$\begin{array}{c}\text { Fillettes } \\
\text { migrantes }\end{array}$} & \multirow{2}{*}{$\begin{array}{l}\text { Fillettes } \\
\text { victimes } \\
\text { de traite }\end{array}$} & \multirow[b]{2}{*}{ Effectif $(N)$} & \multirow[b]{2}{*}{$\%$} \\
\hline & $11-14$ & $15-18$ & & & & \\
\hline Centre de santé & 12 & 22 & 18 & 00 & 52 & 34,66 \\
\hline Guérisseur & 01 & 03 & 02 & 01 & 07 & 04,67 \\
\hline $\begin{array}{c}\text { Automédication } \\
\text { moderne }\end{array}$ & 11 & 13 & 15 & 22 & 61 & 40,67 \\
\hline $\begin{array}{c}\text { Automédication } \\
\text { traditionnelle }\end{array}$ & 04 & 08 & 12 & 06 & 30 & 20,00 \\
\hline $\operatorname{Total}(N)$ & 28 & 46 & 47 & 29 & 150 & 100 \\
\hline
\end{tabular}

Source : Enquête de terrain

Il faut souligner que durant le temps de travail consacré à la vente dans les rues et marchés, la partie des revenus réservée à la nourriture est infime. Dans la journée, les filles se contentent d'un plat d'attiéké ${ }^{36}$ ou de riz accompagné d'un morceau de poisson. A défaut, elles s'alimentent avec tout ce qui est à la portée de leur bourse. Entres autres, on peut ajouter le placali ${ }^{37}$, le cabato ${ }^{38}$, la bouillie (de riz ou de mil), les galettes, pour ne citer que ceuxlà. Etant donné qu'elles passent toute la journée à la vente, cette ration quotidienne varie entre cent cinquante (150) et deux cent cinquante (250) francs CFA ; et ce, en fonction de l'âge des adolescentes. Bien que les données n'aient pas permis de voir la contribution éventuelle de ces adolescentes à la popote, il est probable qu'elles y participent; la manière étant laissée à l'initiative ou à l'appréciation de l'employeur ou de la tutrice.

\footnotetext{
${ }^{36}$ Semoule à base de manioc, qui peut être mangée seule, avec du poisson ou accompagnée d'une sauce. A la portée de toutes les bourses et facile à consommer, l'attiéké est en passe de devenir le met national ivoirien. Il s'exporte aussi dans toute l'Afrique de l'ouest et même en Europe.

${ }^{37}$ Purée de manioc accompagnée d'une sauce.

${ }^{38}$ Purée de maïs accompagnée d'une sauce.
} 
Sur le plan sanitaire, plus de $60 \%$ des petites commerçantes n'a pas accès à un centre de santé. Leur situation sanitaire ne peut donc être reluisante. Dans un tableau globalement sombre sur l'accès de ces adolescentes aux centres de santé, il importe de porter une attention particulière à la situation sanitaire plus catastrophique des fillettes migrantes (seules dix huit parmi toutes celles interrogées ont accès à un centre de santé en cas de pathologie) et celles ayant été victimes de traite (aucune d'entre elles n'a accès à un centre de santé en cas de pathologie). La majorité des fillettes de ces deux groupes n'a recours qu'à l'automédication moderne ou traditionnelle en cas de pathologie. Aussi, le travail dans le secteur informel du petit commerce a des conséquences négatives sur les petites commerçantes. En effet, l'ampleur des accidents et des maladies dont sont victimes les adolescentes qui travaillent est une source de profonde préoccupation. Les adolescentes n'ont pas la même résistance que les adultes, et leur constitution physiologique et psychologique les rend encore plus vulnérables que ceux-ci à certains risques professionnels, dont les effets négatifs sont plus accusés chez elles. N'ayant pas encore assez de maturité, elles sont moins conscientes des risques potentiels inhérents à leurs lieux de travail.

Les conditions de travail dangereuses auxquelles sont soumises les adolescentes peuvent avoir des effets désastreux sur leur santé. Les tâches physiquement pénibles, notamment les manutentions de lourdes charges ou les mauvaises postures de travail, peuvent déformer ou handicaper définitivement un corps en pleine croissance. Un autre type de méfait de l'activité de petit commerce exercée par les adolescentes est lié à la vie à la rue. Ces adolescentes sont exposées à toutes sortes de nuisance et de pollution (bruits et odeurs des automobiles, insalubrité, poussière, odeurs nauséabondes des immondices), aux intempéries (tantôt la chaleur caniculaire, tantôt la pluie). Malheureusement, les adolescentes les plus jeunes sont moins susceptibles de percevoir le danger ou de savoir comment réagir en cas de maladies. Les filles sont souvent victimes de discriminations, et se voit souvent refuser l'accès aux soins de santé. Ce qui nous amène à dire que l'activité de petit commerce exercée par ces adolescentes comporte non seulement des risques de santé pour elles, mais aussi des conséquences pour la société toute entière.

\section{II-4. Brimades et sévices corporels : une série de violences}

Il nous est revenu de façon régulière que le fonctionnement des familles qui emploient des adolescentes dans le secteur informel du petit commerce, se caractérise par sa verticalité. Certains membres, tout particulièrement les employeurs, rassemblent un pouvoir et une autorité reconnus et acceptés par le reste de la famille et en particulier les adolescentes. Le constat est tel que cette configuration familiale engendre le plus souvent un comportement violent de la part de l'employeur avec des conséquences aussi 
bien physiques que psychologiques sur les autres membres de la famille, dont les adolescentes en particulier. En effet, il existe en général, au sein des familles, une violence des adultes sur les adolescentes au travail. Selon notre enquête, à la question « dans votre entourage (voisins, amis, famille), de quelle manière pensez-vous que les pères et mères de famille résolvent généralement leurs problèmes avec leurs enfants adolescents (11 à 18 ans) ? ", près d'un tiers des employeurs interrogées réponde «par les coups » avec des différences une fois de plus notables selon le niveau de vie des couches sociales. On peut donc supposer que les " parents » ou employeurs appartenant à la couche sociale basse tendent davantage à considérer qu'une solution aux problèmes avec les adolescentes qu'elles emploient, se trouve dans les coups, sanctions et punitions ; c'est-à dire par une forme de violence physique et a fortiori psychologique. Dans la même enquête, à la question voisine "Comment pensez-vous que les pères corrigent leurs employées lorsque celles-ci leur manquent de respect ou manquent à leurs obligations ou devoirs ? ", la tendance aux coups ou punitions se confirme nettement et arrive en tête dans plus de $70 \%$ des cas sur les adolescentes; vient ensuite la réponse «par le dialogue » avec moins de $20 \%$.

Dans les relations familiales, le manque de respect ou manquement aux obligations et devoirs est généralement considéré comme la nonreconnaissance de l'autorité ou comme la violation des règles établies. Les adolescentes manifestent alors une protestation contre l'iniquité de pouvoir et la tendance à l'autoritarisme présente dans leur famille où elles travaillent. Le manque de respect d'une adolescente ou son manquement à ses obligations et devoirs peut signifier une réponse disproportionnée à une demande du " parent » ou de l'employeur ou même une violence exercée contre celle-ci de manière physique et/ou verbale. Face à cette "révolte", la réaction du " parent » ou de l'employeur sera la plupart du temps basée sur la violence. Dans cette enquête, la réponse massive attestant l'usage des "coups" et des punitions révèle une tendance nette à la violence comme modalité de résolution des conflits opposant les adolescentes au travail dans le petit commerce à leur « parent » ou employeur. Cette prédisposition se confirme de nouveau dans les réponses à la question « Comment pensez-vous que les «parents» ou employeurs corrigent leurs adolescentes au travail lorsque celles-ci se comportent mal ou n'obéissent pas ? » « en les frappant », réplique violemment une employeuse enquêtée.

Face à de tels constat, l'on peut s'accorder à affirmer que la punition physique est un phénomène très largement répandu au niveau des adolescentes au travail dans le secteur informel du petit commerce lors de leur éducation autant à la ville qu'à la campagne. C'est ainsi que la plupart des petites commerçantes interrogées nous a révélée qu'elles-mêmes se perçoivent comme les membres de leur famille les plus punies. Les punitions sont aussi 
bien données par « la mère » que « le père » ou l'employeur et l'analyse met en exergue une chaîne de violence qui va de l'autorité " des parents » ou de l'employeur, pour aboutir finalement sur les enfants et en priorité sur les adolescentes au travail dans le secteur informel du petit commerce. « Lorsque l'adolescente ou une fille de la famille partage le rôle domestique assigné à la mère, celle-ci se transforme en cible de toute agression. La mère exerce alors la même pression et violence que celle venant de son mari », tels sont les propos recueillis auprès d'un responsable institutionnel en charge de la protection des enfants.

\section{Discussion}

Cette étude, depuis le début, s'est fixée pour objectif principal de contribuer à une meilleure connaissance de l'enfance en danger à partir de la compréhension et de l'explication des conditions de travail et de vie des adolescentes du secteur informel du petit commerce à Abidjan Pour atteindre cet objectif, l'hypothèse suivante a été formulée : Les conditions de travail et de vie difficiles des petites commerçantes dans le secteur informel favorisent chez elles le développement de comportements déviants ou marginaux (recherche de l'argent ou du gain facile) et l'accoutumance aux fléaux modernes (mendicité, prostitution, vol, délinquance, violence, etc.).

Vérifier ces hypothèses suppose que nous discutions des résultats que nous avons obtenus, lesquels nous permettrons de confirmer ou d'infirmer ces hypothèses.

Au total, nous pouvons retenir que les conditions de travail et de vie des adolescentes dans le petit commerce à Abidjan, sont en général difficiles, désastreuses. Elles sont très pénibles à la fois physiquement que psychologiquement. Du coup, leurs impacts sur la santé sont néfastes.

Les adolescentes sont davantage exposées aux accidents professionnels, en raison de l'insécurité des conditions de travail, de l'inexpérience, de la fatigue et aussi du fait que ce sont souvent des activités non déclarées, luttant sur un marché compétitif et instable. D'autres dangers résident dans l'emploi d'objets dangereux et substances toxiques ainsi que dans l'excès de chaleur, de poussière et de bruit, etc.

Toutefois, le fait intéressant qui mérite d'être souligné ici, ce sont les comportements marginaux et la sous-culture par rapport à l'objectif de survie que développent ces fillettes grâce à des stratégies et logiques d'adaptation. Contraintes à se battre pour sortir du cercle infernal de la pauvreté, ces petites commerçantes, essayent tant bien que mal de s'adapter aux dures réalités que leur impose l'exercice du petit commerce. Dans ce milieu dangereux, contraignant et harassant, elles forgent un esprit et un caractère qui non seulement, leur permettent de supporter les souffrances, mais aussi de développer des stratégies qui compenseraient les faibles revenus tirés de leur 
activité principale. Intéressées par les gains, et n’en mesurant pas les dangers, ces petites commerçantes font alors la connaissance de la mendicité, de la violence et l'esprit d'agressivité comme moyen de défense, de la prostitution, de la délinquance et de la consommation de la drogue, avec lesquelles elles entretiennent des rapports étroits.

Cette sous-culture par rapport à l'objectif de survie est une véritable valeur intériorisée par la plupart des adolescentes au travail dans le secteur informel du petit commerce en milieu urbain. C'est elle qui permet à ces adolescentes d'assurer leur conservation personnelle. Et le petit commerce dans les rues d'Abidjan n'est qu'un prétexte qui cache une criminalité astucieuse et certaines formes de déviances.

En tout état de cause, les aspects criminogènes que nous avons évoqués plus haut font partie de la culture de la rue. Dans leur logique de survie dans un monde où elles doivent « se battre » pour surmonter les difficultés, les petites commerçantes font référence en général, à des actes de déviance ou à des valeurs criminelles qui orientent les actes qu'elles posent ou les comportements qu'elles adoptent.

Quoi qu'exposées à la «vie de la rue », sorte de sous-culture qui pourraient les amener à banaliser ou à s'accoutumer aux risques des fléaux modernes que nous avons énuméré, les adolescentes au travail dans le secteur informel du petit commerce, privées de qualification professionnelle, ont foi en l'avenir. Même si le tableau de leur devenir reste sombre, les aspirations de ces jeunes filles traduisent néanmoins leur ténacité et leur volonté de sortir de ce système.

Cette étude confirme également les résultats des recherches antérieurs (Fattah, 1981 \& Mendelsohn, 1973) sur les théories victimologiques. Pour ces auteurs, « Il ne s'agit plus de cristalliser les recherches sur la culpabilité de la victime comme dans la première victimologie, mais de porter un intérêt sur la reconnaissance et le traitement distincts des souffrances subies par les victimes : C'est-à-dire la seconde victimologie. Ces théories ont un rapport certain avec notre objet d'étude du moment où elles mentionnent l'existence d'« éléments communs permettant de découvrir les données générales qui rendent certains individus enclins à devenir des victimes à cause d'un potentiel réduit ou inexistant de résistance, du point de vue biopsychosocial » (Cario cité par Koudou, 2007).

Ainsi, l'adolescente au travail se présentera comme une victime de la réalité sociale; mieux, « une victime totalement innocente » ou 《victime inconsciente » ou encore «victime latente ou prédisposée (victime à prédisposition biopsychologique, socialement prédisposée, moralement ou psychologiquement prédisposée) » (Koudou, 2007). 


\section{Conclusion}

Le travail des enfants est un phénomène complexe dont la dimension économique n'est qu'une composante, probablement la plus importante en Afrique Noire. Contrairement à la situation de nombreuses adolescentes travailleuses en Asie et en Amérique latine, en Côte d'Ivoire, le problème n'est pas fondamentalement une stratégie délibérée de la part des employeurs pour accroître indûment la position compétitive de leur production. De façon générale, il prend ses racines dans la tradition, les contraintes financières, la faiblesse du système éducatif, la structure du marché du travail, les contraintes de compétitivité, etc.

L'environnement global de l'adolescente influence alors son insertion dans des activités socioéconomiques. Ainsi, il apparaît de fortes tensions liées à l'existence de divers choix alternatifs : investissement éducatif, mise en apprentissage, ou utilisation de la main d'œuvre familiale dans l'économie informelle.

Le travail des petites commerçantes, on l'a vu n'est pas l'apanage des pays pauvres, mais c'est toujours la pauvreté qui est la cause principale. Néanmoins, il résulte de multiples facteurs variant suivant les régions du monde et qu'il est essentiel de cerner parfaitement afin de comprendre, non seulement pourquoi les adolescentes travaillent, mais surtout comment faire pour remédier à cette situation.

Nous pensons que c'est la conjugaison de facteurs internes et externes au ménage qui poussent les adolescentes au travail. Ces facteurs doivent être perçus simultanément en ce sens que le travail des adolescentes reste un phénomène social en Côte d'Ivoire. Notre étude révèle également le rôle prépondérant du capital social (lien de parenté et autres normes sociales) dans l'analyse des déterminants du travail exercé par les adolescentes dans le secteur informel du petit commerce. En effet, le travail participe de la vie matérielle aussi bien que de la vie sociale.

Toutefois, le recours à la main d'œuvre infantile ne constitue pas moins un problème grave pour la Côte d'Ivoire. S'il le justifie dans une certaine mesure, il est à signaler qu'il pourrait compromettre le développement physique et mental des petites commerçantes.

Qu'en est-il des pratiques liées aux conditions de travail et de vie des adolescentes dans le secteur informel du petit commerce?

Nous pouvons retenir les conditions de travail et de vie des adolescentes dans le petit commerce à Abidjan, sont en général difficiles, désastreuses. Elles sont très pénibles à la fois physiquement que psychologiquement. Du coup, leurs impacts sur la santé sont néfastes.

Les adolescentes sont davantage exposées aux accidents professionnels, en raison de l'insécurité des conditions de travail, de l'inexpérience, de la fatigue et aussi du fait que ce sont souvent des activités 
non déclarées, luttant sur un marché compétitif et instable. D'autres dangers résident dans l'emploi d'objets dangereux et substances toxiques ainsi que dans l'excès de chaleur, de poussière et de bruit, etc.

Toutefois, le fait intéressant qui mérite d'être souligné ici, ce sont les comportements marginaux et la sous-culture par rapport à l'objectif de survie que développent ces fillettes grâce à des stratégies et logiques d'adaptation. Contraintes à se battre pour sortir du cercle infernal de la pauvreté, ces petites commerçantes, essayent tant bien que mal de s'adapter aux dures réalités que leur impose l'exercice du petit commerce. Dans ce milieu dangereux, contraignant et harassant, elles forgent un esprit et un caractère qui non seulement, leur permettent de supporter les souffrances, mais aussi de développer des stratégies qui compenseraient les faibles revenus tirés de leur activité principale. Intéressées par les gains, et n'en mesurant pas les dangers, ces petites commerçantes font alors la connaissance de la mendicité, de la violence et l'esprit d'agressivité comme moyen de défense, de la prostitution, de la délinquance et de la consommation de la drogue, avec lesquelles elles entretiennent des rapports étroits.

Cette sous-culture par rapport à l'objectif de survie est une véritable valeur intériorisée par la plupart des adolescentes au travail dans le secteur informel du petit commerce en milieu urbain. C'est elle qui permet à ces adolescentes d'assurer leur conservation personnelle. Et le petit commerce dans les rues d'Abidjan n'est qu'un prétexte qui cache une criminalité astucieuse et certaines formes de déviances.

En tout état de cause, les aspects criminogènes que nous avons évoqués plus haut font partie de la culture de la rue. Dans leur logique de survie dans un monde où elles doivent « se battre » pour surmonter les difficultés, les petites commerçantes font référence en général, à des actes de déviance ou à des valeurs criminelles qui orientent les actes qu'elles posent ou les comportements qu'elles adoptent.

Quoi qu'exposées à la «vie de la rue », sorte de sous-culture qui pourraient les amener à banaliser ou à s'accoutumer aux risques des fléaux modernes que nous avons énuméré, les adolescentes au travail dans le secteur informel du petit commerce, privées de qualification professionnelle, ont foi en l'avenir. Même si le tableau de leur devenir reste sombre, les aspirations de ces jeunes filles traduisent néanmoins leur ténacité et leur volonté de sortir de ce système.

\section{References:}

1. AGBADOU, N. J. (2009). Les enfants au travail dans les plantations de café-cacao en Côte d'Ivoire, Abidjan, thèse unique de doctorat non publiée, UFR criminologie, Université cocody. 
2. ANDVIG, J. \& Alii. (2001). Issues on Child Labor in Africa, Africa Region Human Development, Working Paper Series, World Bank.

3. AKTOUF, O. (1987). Méthodologie des sciences sociales et approche qualitative des organisations, une introduction à la démarche classique et une critique. Québec: les Presses de l’Université du Québec.

4. BHUKUTH, A. \& Guérin, I. (2005). Quelle Education pour les Enfants Travailleurs de l'Industrie de la Briqueterie en Inde du Sud, Mondes en Développement, Vol.132

5. Banque Mondiale, Institut National de la statistique (1995). Définition moderne de la pauvreté en Côte d'Ivoire et le profil des pauvres, rapport de travail, Abidjan : BM/INS

6. BAZZI-VEIL, L.et al. (2003). Analyse de la situation du travail des enfants en Côte d'Ivoire, Abidjan, CEPRASS/UNICEF, novembre.

7. BIT/Projet/WACAP. (2003). Signature du mémorandum d'accord. Abidjan

8. BIT. (2005). La traite des enfants aux fins d'exploitation de leur travail dans le secteur informel à Abidjan, rapport d'enquête, Côte d'Ivoire.

9. BOUCHET-SAULNIER. (2006). Dictionnaire pratique du droit humanitaire .

10. Convention Relative aux Droits de l'Enfant. (1989). adoptée par les Nations Unies le 29 novembre.

11. COHEN, L, E et FELSON, M. (1979), « Social change and crime rate trends. A routine activity approach », American sociological review, Pp 588-608.

12. DIALLO, Y. \& Koné, S. (2001). Pauvreté des ménages et phénomène du travail des enfants en Côte d'Ivoire, Université Montesquieu-Bordeaux IV.

13. DUMAS, C. (2005). Offre de travail des enfants et demande d'éducation dans les pays d'Afrique de l'Ouest, Thèse de Doctorat, Ecole des hautes études en sciences sociales.

14. FATTAH, E, A. (1981). «La victimologie entre les critiques épistémologiques et les attaques idéologiques », Dév. et soc. Pp 71 $-92$.

15. FONCHINGONG, C, C. (2005). Au-delà de Beijing, l'exemple d'une stratégie de survie: l'existence difficile des marchandes d'aliments du secteur informel à Limbé (Cameroun), in Revue internationale des sciences sociales, Eres, $\mathrm{n}^{\circ} 184$, Fenouillet (France). 
16. GRAWITZ, M. (2008). Lexique des sciences sociales, $8^{\text {ème }}$ Edit.

17. Institut National de la Statistique. (2008). Enquête sur le Niveau de Vie des Ménages, p.7.

18. Institut National de Statistique. (2005). Enquête nationale sur le travail des enfants. Abidjan : Ministère de la Fonction Publique et de l'Emploi - BIT-IPEC - INS

19. JACQUEMIN, M. (2002). Travail domestique et travail des enfants, le cas d'Abidjan (Côte d'Ivoire), in Tiers Monde, n¹70, PUF, Paris, France.

20. KONE, M, P. (2014). Condition de travail et de vie des enfants immigrés à Abidjan. Mémoire de DEA, UFR criminologie non publié, Université Félix Houphouët Boigny.

21. KOUAKOU, K. (2006). L'Exploitation des enfants dans le contexte de la crise militaire et politique en Côte d'Ivoire : Situation particulière dans les zones d'accueil des déplacés et des réfugiés, BIT -IPEC/LUTRENA, UNHCR, Dakar.

22. KOUDOU, O. (2007). Histoire de la criminologie : Les grands courants théoriques d'hier à aujourd'hui, PUA, P 107.

23. KODZOVI, S, A. (2011). Etude comparative des conditions de travail des enfants issus des ménages agricoles au Burkina-Faso, en Côte d'Ivoire et au Mali, Ecole nationale et supérieure de statistiques et d'économie d'Abidjan - Ingénieur statisticien.

24. LACHAUD, P. (2004). Le travail des enfants et la pauvreté en Afrique : Un réexamen appliqué au Burkina Faso, Université Montesquieu - Bordeaux IV.

25. MENDELSOHN, B. (1973), « La victimologie et les besoins de la société actuelle », R.I.C.P.T., Pp $267-276$.

26. Ministère d'ETAT, Ministère du Plan et du Développement de la République de Côte d'Ivoire. (2006). Côte d'Ivoire, suivie de la situation des enfants et des femmes, enquête par grappes à indicateurs multiples.

27. Nation Unies, Convention des Nations contre la criminalité transnationale organisée. (2001).Assemblée générale, $\mathrm{A} / \mathrm{RES} / 55 / 25, \quad 55^{\mathrm{e}}$ session, http://www.unodc.org/pdf/crime/a_res_55/res5525£pdf.

28. MUCHIELLI, R. (1965). Comment ils deviennent délinquants. Genèse et développement de la socialisation et de la dissocialité, éd. Sociales françaises, Paris

29. N'DA, P. (2015). Méthodologie de la recherche. De la problématique à la discussion des résultats. Comment réaliser un mémoire, une thèse, en science sociale et en éducation, Abidjan : EDUCI. 
30. OIT. Conventions $\mathrm{n}^{\circ} 138$ et 182 relative à l'âge minimum d'admission à l'emploi.

31. OIT/BIT. (2000). Convention $n^{\circ} 182$ relative aux pires formes du travail des enfants et l'action immédiate en vue de leur élimination, adoptée à GENEVE le 17 juin 1999.

32. PNUD. (2010). Indice de la pauvreté multidimensionnelle.

33. QUIVY, R. et CAMPENDOUT. L. (1995). Manuel de recherche en sciences sociales, Paris, Dunod, $2^{\text {ème }}$ Edit

34. SCHLEMMER, B. (1997). L'exploitation des enfants au travail : d'un problème social à une problématique en science sociale, cahier de Marjuvia ${ }^{\circ} 10$.

35. SOMMARUGA PHILLOT, $S$ et alii (2007), Travail des enfants dans le monde : un problème de société, in Revue Médicale Suisse, éd. Médecine et Hygiène, vol. 3, $\mathrm{n}^{\circ}$ 100, Genève (Suisse), pp. 554556.

36. UNICEF. (2002). La situation des enfants dans le monde, prendre l'initiative, Genève, Unicef Housse.

37. VOELIN, C et alii (2006), Un observatoire de la maltraitance des enfants : une façon interactive de s'intéresser à cette problématique, in Revue Médicale Suisse, éd. Médecine et Hygiène, vol. 2, n 54, Genève (Suisse), pp. 538-541. 